\title{
DEGRADAÇÃO FOTOCATALÍTICA DO FUNGICIDA TEBUCONAZOLE EM SOLUÇÃO AQUOSA
}

\author{
Thiago de Hermann Prestes, Danielle de Oliveira Gibbon e Marla Azário Lansarin*
}

Departamento de Engenharia Química, Universidade Federal do Rio Grande do Sul, Rua Luiz Englert, s/n, $90040-040$ Porto

Alegre - RS, Brasil

Celso Camilo Moro

Departamento de Química Inorgânica, Instituto de Química, Universidade Federal do Rio Grande do Sul, CP 15003, 91501-970

Porto Alegre - RS, Brasil

Recebido em 16/4/09; aceito em 3/11/09; publicado na web em 24/3/10

\begin{abstract}
TEBUCONAZOLE PHOTOCATALYTIC DEGRADATION KINETICS. The tebuconazole photocatalytic degradation kinetics was studied in a batch reactor using $\mathrm{TiO}_{2}$ (P25-Degussa) as catalyst and a high pressure mercury lamp. The photolysis, adsorption and irradiation effects in the reaction rate were evaluated. Afterward, the suspension catalyst concentration and initial $\mathrm{pH}$ to the maximum reaction rate was determined. It was observed that the reaction rate can be approached by a pseudo-first order, with a maximum kinetics constant at $260 \mathrm{mg} \mathrm{L}^{-1}$ catalyst concentration and $\mathrm{pH} 7.7$.
\end{abstract}

Keywords: tebuconazole; photocatalysis; kinetics.

\section{INTRODUÇÃO}

A contaminação dos mananciais de água por moléculas orgânicas potencialmente tóxicas é um problema ambiental que tem se agravado nos últimos anos. ${ }^{1,2}$ Dentre estes contaminantes encontra-se o tebuconazole, um fungicida amplamente usado na agricultura, principalmente de cereais. ${ }^{3}$ Este fungicida pode causar danos à saúde humana, podendo provocar irritação cutânea, ocular e respiratória; além de ser tóxico a micro-organismos aquáticos, algas e peixes. ${ }^{4}$

Foram encontrados na literatura alguns trabalhos sobre a degradação fotocatalítica de fungicidas, ${ }^{5,6}$ mas, especificamente sobre a degradação fotocatalítica do tebuconazole, as referências são escassas. Calza et al. ${ }^{3}$ realizaram um estudo visando identificar os produtos e os intermediários formados durante a degradação fotocatalítica do tebuconazole em presença de dióxido de titânio. Concluíram que o tebuconazole é degradado por meio de três rotas diferentes, levando à formação de diversos intermediários, todos eles mais hidrofílicos que o composto original. Não foi realizado um estudo cinético ou um levantamento da influência das variáveis de operação na reação.

Existem, ainda, trabalhos que relatam o emprego de processos oxidativos avançados na degradação do herbicida atrazina, ${ }^{7}$ do bisfenol $\mathrm{A},{ }^{8}$ de anti-inflamatórios não esteroidais, como ibuprofeno e diclofenaco, ${ }^{9,10}$ e de antibióticos, como tetraciclina e sulfametoxazol e derivados da fluoroquinolona. ${ }^{11-14}$ Melo e colaboradores, ${ }^{15}$ por exemplo, apresentaram uma revisão da literatura sobre a ocorrência registrada de fármacos em ambientes aquáticos, desde águas potáveis até estações de tratamento de esgotos, e abordaram as diferentes técnicas empregadas para a eliminação desses compostos, dando ênfase aos processos oxidativos avançados (POAs). Segundo os autores, apesar da complexidade dessas moléculas, os POAs apresentam alta eficiência na sua degradação.

O presente trabalho é parte de um projeto cujo objetivo é verificar a viabilidade técnica e econômica da degradação fotocatalítica do tebuconazole presente em efluentes aquosos. Neste documento estudase a cinética da degradação fotocatalítica deste fungicida em solução aquosa, usando-se um reator do tipo slurry e $\mathrm{TiO}_{2}$ como catalisador.

\footnotetext{
*e-mail: marla@enq.ufrgs.br
}

\section{PARTE EXPERIMENTAL}

\section{Materiais e reagentes}

Os ensaios foram realizados em um reator slurry, cilíndrico, de vidro, com capacidade de $1000 \mathrm{~mL}$, encamisado, recoberto por folha de alumínio e acondicionado em uma caixa fechada provida de ventilador. A água da camisa foi mantida a temperatura constante $\left(30^{\circ} \mathrm{C}\right.$ ) por meio de um banho termostático de $40 \mathrm{~L}$ (Haake DC 30 ). $\mathrm{O}$ monitoramento da temperatura foi feito usando-se um termopar do tipo $\mathrm{K}$ imerso na solução. O reator foi agitado por meio de um agitador magnético e um borbulhador de ar foi colocado no fundo para garantir que houvesse oxigênio no meio. A fonte de irradiação foi uma lâmpada de vapor de mercúrio de alta pressão Philips, com $125 \mathrm{~W}$ de potência, cujo bulbo foi modificado de modo a permitir a passagem de radiação acima de $350 \mathrm{~nm}$. Como catalisador foi utilizado $\mathrm{TiO}_{2}$ (Degussa P-25, 80\% anatase $-20 \%$ rutilo, área superficial de $\left.50 \mathrm{~m}^{2} \mathrm{~g}^{-1}\right)$ sem tratamento prévio.

O tebuconazole usado para preparar as soluções aquosas foi fornecido pela empresa Milenia Agrociências S.A. O reagente é encontrado na forma sólida com $99,7 \%$ de pureza. As soluções foram preparadas com concentração de $30 \mathrm{mg} \mathrm{L}^{-1}$ e o volume total da mistura reacional foi de $900 \mathrm{~mL}$.

\section{Metodologia}

Após ser carregado, o reator era mantido sob agitação na ausência de irradiação por $60 \mathrm{~min}$, de modo a se atingir o equilíbrio de adsorção do tebuconazole na superfície do catalisador. Antes de começar a reação, era medida a irradiação incidente na superfície do líquido. Para isso se utilizou um radiômetro Cole Parmer modelo 9811-50 equipado com um sensor que mede a irradiação no comprimento de onda de $365 \mathrm{~nm}$. A lâmpada era mantida acesa por 3 min antes de iniciar a reação, para atingir o máximo de irradiação. $\mathrm{O}$ pH da mistura reacional era monitorado em três etapas distintas: na solução de tebuconazole antes de adicionar o catalisador, na mistura reacional após a adição de catalisador e ao final da reação.

Durante os experimentos, amostras de 10 ou $7 \mathrm{~mL}$ (para amostras em triplicata ou duplicata, respectivamente) eram coletadas em 
intervalos de 10 ou 20 min, através de um sistema de seringa-cateter. As amostras eram diluídas na proporção 1:3, centrifugadas em uma centrífuga Centribio por 20 min e a fase sobrenadante era centrifugada por mais 20 min, para garantir a máxima remoção possível do dióxido de titânio. Em todos os ensaios, a variação máxima do volume reacional foi de $90 \mathrm{~mL}$, podendo ser ignorada.

Após as centrifugações, a amostra era analisada no espectofotômetro Varian Carry 100 no comprimento de onda de 197 nm, por ser o de máxima absorbância para o tebuconazole. Foi construída uma curva de trabalho que relaciona a absorbância com a concentração de reagente (coeficiente de correlação, $\mathrm{R}^{2}$, de 0,9997 ). Todos os experimentos foram realizados, no mínimo, em duplicata.

A influência da fotólise foi analisada somente na presença da radiação UV (sem catalisador), no mesmo aparato experimental descrito anteriormente. Foram retiradas amostras do reator durante $2 \mathrm{~h}$ em intervalos de $10 \mathrm{~min}$.

Foi investigado o tempo que o poluente leva para atingir o equilíbrio de adsorção-dessorção na superfície do catalisador. Os ensaios foram realizados no reator slurry, com borbulhamento de ar e 600 $\mathrm{mg} \mathrm{L}^{-1}$ de concentração de catalisador, na ausência de irradiação. As amostras foram coletadas nos tempos 0, 20, 40, 70 e 100 min, centrifugadas imediatamente e analisadas.

Para determinar a influência da fonte de irradiação, foram feitas reações fotocatalíticas no mesmo aparato experimental pelo período de $2 \mathrm{~h}$ (precedidas por $1 \mathrm{~h}$ de escuro), mantendo-se a concentração de catalisador fixa em $200 \mathrm{mg} \mathrm{L}^{-1}$ e o pH natural da solução $(5,5)$. A variação da intensidade de radiação foi feita alterando-se a distância entre a fonte e a solução. Foram realizados experimentos com 0,7 ; 1,$1 ; 1,4 ; 1,8 ; 2,3$ e $3,0 \mathrm{~mW} \mathrm{~cm}{ }^{-2}$.

\section{RESULTADOS E DISCUSSÃO}

Partiu-se da hipótese que a reação obedeceria a uma cinética de pseudoprimeira ordem. Para verificar a veracidade desta hipótese, construiu-se a Figura 1. A boa linearização (representada por um alto valor de $\mathrm{R}^{2}$ ) e o fato dela cruzar a origem indicam que esta hipótese é verdadeira e, assim sendo, a constante cinética aparente passou a ser usada para comparar os diferentes experimentos entre si.

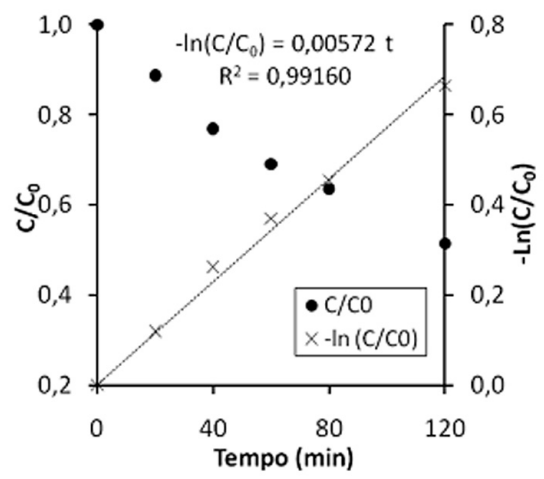

Figura 1. Reação de degradação de tebuconazole. No eixo principal (à esquerda), $C / C_{0}$. No eixo secundário (à direita), $-\ln \left(C / C_{0}\right.$ ). Irradiação $=3,0 \mathrm{~mW} / \mathrm{cm}^{2}$, $p H$ inicial $=5,5$, concentração de catalisador $=200 \mathrm{mg} \mathrm{L}^{-1}, C_{0}=30 \mathrm{mg} \mathrm{L}^{-1}$

\section{Ensaios preliminares}

\section{Efeito da fotólise}

A contribuição da fotólise para a degradação total do poluente foi investigada. Para tanto, a Figura 2 foi construída, permitindo comparar a variação da concentração do poluente ao longo do tempo em uma reação fotocatalítica e uma reação puramente fotolítica. Nesta figura pode-se observar que a fotólise é desprezível frente à reação fotocatalítica, por ser muito mais lenta.

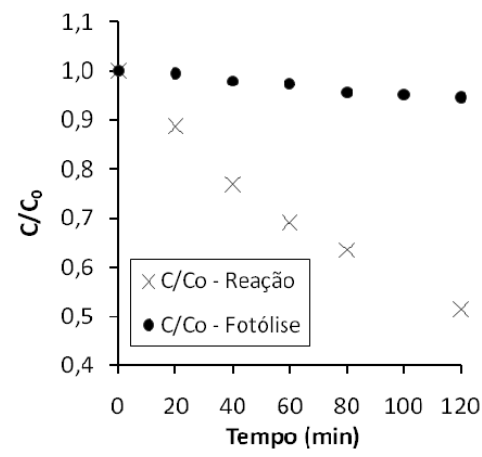

Figura 2. Reação de degradação de tebuconazole. Irradiação $=3,0 \mathrm{~mW} / \mathrm{cm}^{2}$, $p H=$ inicial 5,5, concentração de catalisador $=200 \mathrm{mg} \mathrm{L}^{-1}, C_{0}=30 \mathrm{mg} \mathrm{L}^{-1}$

Teste de adsorção

O tempo necessário para que ocorra o equilíbrio de adsorçãodessorção na superfície do catalisador deve ser conhecido, pois a variação da concentração devido à adsorção pode ser confundida com degradação. Foi realizado um ensaio, tendo-se observado que o equilíbrio é atingido em menos de $40 \mathrm{~min}$. Mesmo assim, adotou-se o tempo de $1 \mathrm{~h}$ para o contato entre catalisador e poluente, no escuro, em todos os experimentos. A concentração de equilíbrio da solução de tebuconazole foi determinada e tomada como a concentração inicial da substância para a análise cinética.

\section{Ensaio de longa duração}

Para verificar se é possível alcançar a degradação total do tebuconazole, foi realizado um ensaio de longa duração. Ao final de $5 \mathrm{~h}$ de reação alcançou-se uma conversão maior que $90 \%$ do reagente. Apesar de a degradação desta molécula ocorrer através de três rotas paralelas, envolvendo um grande número de intermediários, ${ }^{3}$ ao término do ensaio nenhum deles foi detectado, comprovando a eficácia do processo para a purificação deste tipo de efluente, como pode ser visto na Figura 3.

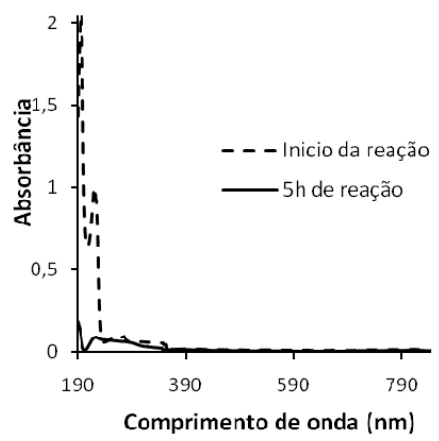

Figura 3. Acompanhamento da concentração do tebuconazole em um ensaio de longa duração. Irradiação $=3,0 \mathrm{~mW} / \mathrm{cm}^{2}, \mathrm{pH}=7,7$, concentração de catalisador $=230 \mathrm{mg} \mathrm{L}^{-1}, C_{0}=30 \mathrm{mg} \mathrm{L}^{-1}$

\section{Influência da irradiação}

Os resultados obtidos estão reunidos na Figura 4. Observa-se uma tendência aproximadamente linear para a constante aparente de taxa quando se varia a irradiação entre 0,7 e $3,0 \mathrm{~mW} \mathrm{~cm}^{-2}$. Este resultado vai ao encontro de Herrmann, ${ }^{16,17}$ segundo a qual a taxa da reação varia linearmente com a irradiação (I) até $25 \mathrm{~mW} \mathrm{~cm}^{-2} \mathrm{e}$, a partir deste 
ponto, varia proporcionalmente com a raiz quadrada da irradiação $\left(\mathrm{k} \alpha \mathrm{I}^{0,5}\right)$. Gogate e Pandit ${ }^{18}$ acrescentam que a variação deixa de ser linear para um determinado valor de radiação, o qual depende das condições do reator e da natureza do efluente.

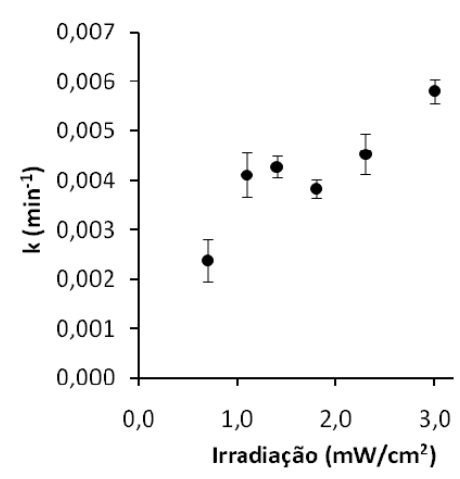

Figura 4. Efeito da irradiação na constante cinética aparente ( $k$ ). pH inicial $=5,5$, concentração de catalisador $=200 \mathrm{mg} \mathrm{L}^{-1}, C_{0}=30 \mathrm{mg} \mathrm{L}^{-1}$

\section{Influência da concentração de catalisador}

Para determinar a influência da concentração de catalisador na mistura reacional sobre a velocidade da reação, foram realizados ensaios, no mesmo aparato experimental, mantendo-se o fluxo de irradiação em $3,0 \mathrm{~mW} \mathrm{~cm}^{-2} \mathrm{e} \mathrm{o} \mathrm{pH}$ natural da solução $(5,5)$, pelo período de $1 \mathrm{~h}$ (precedidas por $1 \mathrm{~h}$ de escuro). As amostras foram coletadas em triplicata, nos tempos 0, 10, 20, 40 e 60 min. Foram realizados experimentos entre 0,0 (fotólise) e $450,0 \mathrm{mg} \mathrm{L}^{-1}$ de fotocatalisador. Os resultados são apresentados na Figura 5, onde se observa um máximo para a concentração de $260 \mathrm{mg} \mathrm{L}^{-1}$.

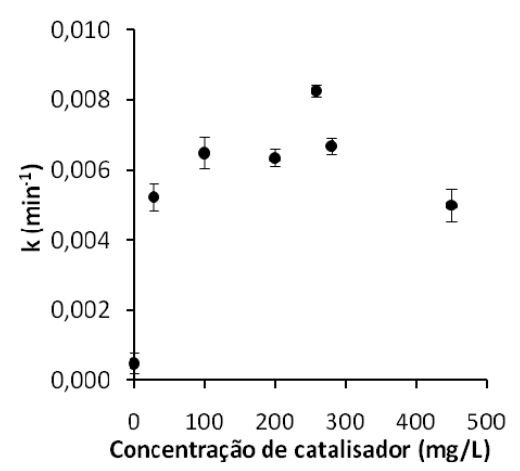

Figura 5. Efeito da concentração de catalisador na constante cinética aparente ( $k$ ). Irradiação $=3,0 \mathrm{~mW} \mathrm{~cm} \mathrm{~cm}^{-2}, \mathrm{pH}$ inicial 5,5, $C_{0}=30 \mathrm{mg} \mathrm{L}^{-1}$

De acordo com Gogate e Pandit, ${ }^{18} \mathrm{o}$ aumento da concentração de catalisador gera um crescimento da quantidade de partículas irradiadas e, consequentemente, da taxa de geração de elétrons livres, até que se alcance uma concentração crítica, onde todas as partículas estão iluminadas. A partir deste ponto, o incremento da concentração de catalisador aumenta a opacidade do sistema reacional, ocasionando um maior espalhamento e diminuição da penetração da luz na solução, levando a uma queda na eficiência do processo. Ainda segundo os mesmos autores, o ponto de máxima taxa de reação irá depender de fatores como condições operacionais, natureza e concentração do poluente.

Outros autores (Silva, ${ }^{19}$ Soares $^{20}$ e $^{\text {Taffarel }}{ }^{21}$ ) usaram o mesmo aparato experimental para determinar a concentração de catalisador para a qual a taxa da reação de fotodegradação é máxima $\left(\mathrm{C}_{\max }\right)$. Sil- va, ${ }^{19}$ trabalhando com fenol com concentrações iniciais de $50 \mathrm{mg} \mathrm{L}^{-1}$, verificou que $300 \mathrm{mg} \mathrm{L}^{-1}$ de catalisador na suspensão propiciam um valor máximo para a constante aparente de taxa. Um valor, portanto, muito próximo do encontrado no presente trabalho $\left(260 \mathrm{mg} \mathrm{L}^{-1}\right)$, onde a concentração inicial de poluente foi de, aproximadamente, $30 \mathrm{mg}$ $\mathrm{L}^{-1}$. No entanto, Soares ${ }^{20}$ (trabalhando com rodamina B) e Taffare ${ }^{21}$ (trabalhando com estireno) encontraram valores de 650 e $550 \mathrm{mg} \mathrm{L}^{-1}$ para este mesmo valor. Taffarel usou concentrações iniciais de estireno entre 24 e $34 \mathrm{mg} \mathrm{L}^{-1}$ e Soares usou concentrações em torno de $20 \mathrm{mg}$ $\mathrm{L}^{-1}$. Em todos estes trabalhos, empregaram-se condições operacionais semelhantes e concentrações iniciais de poluente próximas, tendo-se obtido resultados discrepantes para $\mathrm{C}_{\max }$. Portanto, como mencionado na literatura, a natureza do poluente exerce forte influência em $\mathrm{C}_{\max }$.

\section{Influência do pH inicial da mistura reacional}

Observou-se que o $\mathrm{pH}$ da mistura reacional varia ao longo do experimento, tornando-se cada vez mais ácido (sempre menor do que 5 em todas as reações). Como não havia interesse em controlar (manter constante) esta variável ao longo dos ensaios, optou-se por determinar a influência do $\mathrm{pH}$ inicial da mistura reacional, o que permitiria que se realizasse um único ajuste desta variável, após a adição do catalisador, antes de ligar a lâmpada. Assim, foram feitas reações fotocatalíticas no mesmo aparato experimental, mantendo-se o fluxo de irradiação em $3,0 \mathrm{~mW} \mathrm{~cm}^{-2} \mathrm{e}$ a massa de catalisador em $200 \mathrm{mg} \mathrm{L}^{-1}$, pelo período de $1 \mathrm{~h}$ (precedidas por $1 \mathrm{~h}$ de escuro). As amostras foram coletadas em triplicata, nos tempos 0, 10, 20, 40 e $60 \mathrm{~min}$. Foram realizados experimentos com pH inicial entre 5,2 e 8,9. Os resultados são apresentados na Figura 6, onde se observa um máximo para pH 7,7.

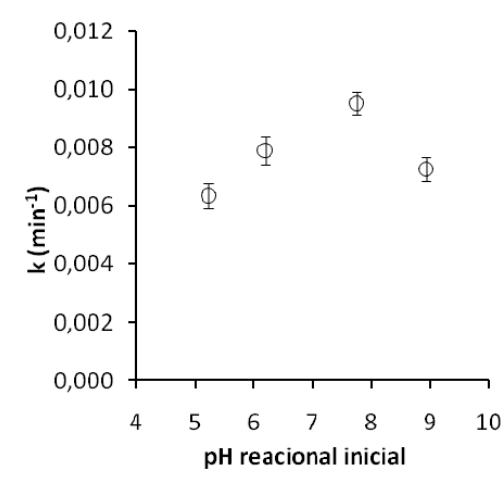

Figura 6. Efeito do pH inicial da mistura reacional na constante cinética aparente ( $k$ ). Irradiação $=3,0 \mathrm{~mW} \mathrm{~cm}^{-2}$, massa de catalisador $=200 \mathrm{mg} \mathrm{L}^{-1}$, $C_{0}=30 \mathrm{mg} \mathrm{L}^{-1}$

Gogate e Pandit ${ }^{18}$ afirmam que o efeito do $\mathrm{pH}$ do meio na taxa da oxidação fotocatalítica é complexo e depende do poluente e do potencial de carga zero do catalisador; mais especificamente, da interação eletrostática entre a superfície do catalisador e o poluente. Segundo os mesmo autores, a adsorção e a taxa da reação serão máximas próximo ao ponto de carga zero, 6,8 para dióxido de titânio, o que também foi determinado por Konstantinou e Albanis. ${ }^{22}$

$\mathrm{O}$ pH para a máxima taxa de reação é próximo ao encontrado por outros autores, ${ }^{20,21}$ que usaram o mesmo aparato experimental na degradação de outras moléculas com o mesmo catalisador.

\section{Influência da temperatura}

A influência da temperatura foi investigada fazendo-se testes nas condições de máxima taxa, variando-se a temperatura de 23 a $40{ }^{\circ} \mathrm{C}$. Observa-se, na Figura 7, que os ensaios realizados a 23 e 
$40{ }^{\circ} \mathrm{C}$ apresentam resultados significativamente diferentes, sendo possível calcular a energia de ativação: $4 \mathrm{kcal} \mathrm{mol}^{-1}\left(16,7 \mathrm{~kJ} \mathrm{~mol}^{-1}\right)$. Herrmann ${ }^{17}$ afirma que a energia de ativação das reações fotocatalíticas é nula, enquanto que a energia de ativação aparente é, para temperaturas entre 20 e $80{ }^{\circ} \mathrm{C}$, de poucos $\mathrm{kJ} \mathrm{mol}^{-1}$. Segundo a autora, para temperaturas acima de $70-80{ }^{\circ} \mathrm{C}$ a energia de ativação aparente torna-se negativa, comportamento compatível com o mecanismo de Langmuir-Hinshelwood.

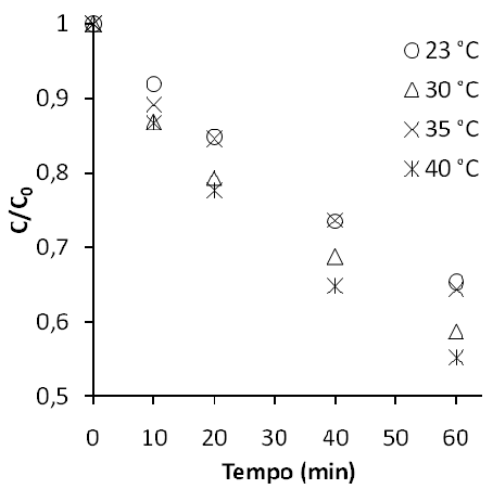

Figura 7. Efeito da temperatura na reação de degradação. Irradiação $=3,0$ $m W \mathrm{~cm}^{-2}, \mathrm{pH}$ inicial $=7,7$, concentração de catalisador $=260 \mathrm{mg} \mathrm{L}^{-1}, C_{0}$ $=30 \mathrm{mg} \mathrm{L}^{-1}$

\section{CONCLUSÕES}

Os resultados obtidos neste trabalho permitem concluir que a degradação fotocatalítica do tebuconazole obedece a uma cinética de pseudoprimeira ordem. Variando-se a intensidade de irradiação entre 0,7 e $3,0 \mathrm{~mW} \mathrm{~cm}^{-2}$ observa-se um crescimento proporcional na velocidade da reação. Por outro lado, ao aumentar gradativamente a concentração de catalisador na suspensão, encontra-se um máximo em $260 \mathrm{mg} \mathrm{L}^{-1}$. Também existe um valor de $\mathrm{pH}$ inicial da solução para o qual a velocidade de reação é máxima: 7,7 nas condições empregadas. Além disso, verificou-se que a temperatura pouco influencia a velocidade da reação, uma vez que a energia de ativação é muito baixa.

Observou-se, ainda, que a degradação do tebuconazole por fotólise pode ser negligenciada, uma vez que é muito mais lenta que a reação fotocatalisada. O equilíbrio de adsorção é atingido em aproximadamente $40 \mathrm{~min}$, quando se trabalha com uma concentração de catalisador de $600 \mathrm{mg} \mathrm{L}^{-1}$ e concentração inicial de tebuconazole na solução de $30 \mathrm{mg} \mathrm{L}^{-1}$ e temperatura de $30^{\circ} \mathrm{C}$.

\section{AGRADECIMENTOS}

À Capes pelo apoio financeiro. À Degussa pelo fornecimento do catalisador. À Milenia Agrociências S.A. pelo reagente.

\section{REFERÊNCIAS}

1. Khetan, S. K.; Collins, T. J.; Chem. Rev. 2007, 107, 2319.

2. Billa, D. M.; Dezotti, M.; Quim. Nova 2007, 30, 651.

3. Calza, P.; Baudino, S.; Aigotti, R.; Baiocchi, C.; Branca, P.; Pelizzetti, E.; J. Mass Spectrom. 2002, 37, 566.

4. http://www.milenia.com.br, acessado em Outubro 2007.

5. Lhome, L.; Brosillon, S; Wolbert, D.; J. Photochem. 2007, 188, 34.

6. Ishiki, R. R.; Ishiki, H. M.; Takashima, K.; Chemosphere 2005, 58, 1461.

7. Santana, H.; Bonancêa, C. E.; Takashima, K.; Quim. Nova 2003, 26, 807.

8. Gómez, L. F.; Sarriá, V. M.; Quim. Nova 2009, 32, 1164.

9. Méndez-Arriaga, F.; Esplugas, S.; Giménez, J.; Water Res. 2008, 42, 585 .

10. Rizzo, L.; Meric, S.; Kassinos, D.; Guida, M.; Russo, F.; Belgiorno, V.; Water Res. 2009, 43, 979.

11. Palominos, R. A.; Mondaca, M. A.; Giraldo, A.; Peñuela, G.; PérezMoya, M.; Mansilla, H. D.; Catal. Today 2009, 144, 100.

12. Trovó, A. G.; Nogueira, R. F. P.; Agüera, A.; Fernandez-Alba, A. R.; Sirtori, C.; Malato, S.; Water Res. 2009, 43, 3922.

13. Paul, T.; Miller, P. L.; Strathmann, T. J.; Environ. Sci. Technol. 2007, 41, 4720.

14. Reyes, C.; Fernández, J.; Freer, J.; Mondaca, M. A.; Zaror, C.; Malato, S.; Mansilla, H. D.; J. Photochem. Photobiol. A Chem. 2006, 184, 141.

15. Melo, S. A. S.; Trovó, A. G.; Bautitz, I. R.; Nogueira, R. F. P.; Quim. Nova 2009, 32, 188.

16. Hermann, J.; Catalysis Today 1999, 53, 115.

17. Hermann, J.;Topics in Catalysis 2005, 34, 49.

18. Gogate, P. R.; Pandit, A. B.; Adv. Env. Res. 2004, 8, 501.

19. Silva, F. V.; Dissertação de Mestrado, Universidade Federal do Rio Grande do Sul, Brasil, 2007.

20. Soares, E. T.; Lansarin, M. A.; Moro, C. C.; Braz. J. Chem. Eng. 2007, $24,29$.

21. Taffarel, S. R.; Dissertação de Mestrado, Universidade Federal do Rio Grande do Sul, Brasil, 2007.

22. Konstantinou, I. K.; Albanis, T. A.; Appl. Catal. B: Environ. 2004, 49, 1. 